\title{
VALUE-AT-RISK AND EXPECTED SHORTFALL FOR LINEAR PORTFOLIOS WITH ELLIPTICALLY DISTRIBUTED RISK FACTORS
}

\author{
JULES SADEFO KAMDEM \\ LABORATOIRE DE MATHÉMATIQUE \\ CNRS UMR 6056 \\ UNIVERSITÉ DE REIMS
}

\begin{abstract}
In this paper, we generalize the parametric $\Delta$-VaR method from portfolios with normally distributed risk factors to portfolios with elliptically distributed ones. We treat both the expected shortfall and the Value-at-Risk of such portfolios. Special attention is given to the particular case of a multivariate $t$-distribution.
\end{abstract}

Key Words: Elliptic distributions, linear portfolio, Value-at-Risk, Expected Shortfall, capital allocation.

\section{Introduction}

The original RiskMetrics methodology for estimating VaR was based on parametric methods, and used the multi-variate normal distribution. This approach works well so-called linear portfolios, that is, those portfolios whose aggregate return is, to a good approximation, a linear function of the returns of the individual assets which make up the portfolio, and in situations where the latter can be assumed to be jointly normally distributed. For other portfolios, like portfolios of derivatives depending non-linearly on the return of the underlying, or portfolios of non-normally distributed assets, one generally turns to Monte Carlo methods to estimate the VaR. Monte Carlo methodology has the obvious advantage of being almost universally applicable, but has the disadvantage of being much slower than comparable parametric methods, when the latter are available. This is an issue in situations demanding for real-time evaluation of financial risk. For nonlinear portfolios, practitioners, as an alternative to Monte Carlo, use $\Delta$-normal VaR methodology, in which the portfolio return is linearly approximated, and an assumption of normality is made. Such methods present us with a trade-off between accuracy and speed, in the sense that they are much faster than Monte-carlo, but much less accurate unless the linear approximation is quite good, and the normality hypothesis holds well. In case the linear approximation is of poor quality, or inherently instable (as is for example the case when the portfolio $\Delta$ is close to 0 ), one turns to a higher order approximations, for example the quadratic one, while keeping the normality assumption. This leads to the so-called $\Gamma-\Delta$ VaR, which

This draft is a part of J.SADEFO-KAMDEM (university of Reims)PhD Thesis. It has been presented at the workshop on modelling and computation in Financial Engineering at Bad Herrenalb, Germany May 6-8,2003.

Many thanks for the comments of Professor R.Brummelhuis.

Author's address: University of Reims, Laboratoire de Mathématique UMR 6056-CNRS , BP 1039 Moulin de la Housse, 51687 Reims cedex 2 FRANCE.

e-mail: sadefo@univ-reims.fr. 
can be evaluated using Mont Carlo, but for which also a number of semi-parametric methods have been developed (see for example Duffy and Pan [2], and others.

An obvious first generalization is to keep the linearity assumption, but replace the normal distribution by some other family of multi-variate distributions. This is the subject of the present paper. As an alternative to the normal hypothesis we will assume that the log returns of the portfolio's constituents have a multivariate elliptic distribution in one of the elliptic classes $N(\mu, \Sigma, \phi)$, cf. section 1 below for the precise definition. These have the advantage that,like normal distributions, their dependence structure is completely determined by their mean and variance, once a choice is made for $\phi$. Particular examples are the normal distributions and the multi-variant Student- $t$ distributions. The latter are an obvious first choice since they posses heavy tails.

Glasserman, Heidelberger and Shahabuddin [5] present a method to compute even $\Gamma-\Delta$ VaR using a semi-parametric method based on the Fourier transform, but their methodology seems to be restricted to $t$-distributions. See also Lopez and Walter [8] and references therein for further applications of the $t$ distribution to VaR. Note that one shortcoming of the multivariate t-distribution is that all the marginal distributions must have the same degrees of freedom, which implies that every risk factor has equally heavy tails.

In a sequel paper we intend to extend the present analysis to include quadratically non-linear portfolios. We note that in a companion paper we give very precise analytic VaR estimates for the VaR of such quadratical portfolios, in case the underlying risk-factors follow another class of non-Gaussian distributions, namely the multivariate generalized normal distributions.

The paper is organized, as follows: In section 2, we will analyze the VaR of a linear portfolio with elliptically distributed risk-factors, paying special attention to the case of a multi-variate Student distribution. Used in conjunction with a first order Taylor approximation of a portfolio's Profit \& Loss function, this will give rise to the notions of Delta-Elliptic VaR and Delta-Student VaR, in analogy with the familiar Delta-Normal VaR. We show, for example, how to reduce the computation of the Delta-Student VaR to finding the zero's of a certain special function. In section 3 we show how to extend our procedure to mixtures of elliptic distributions. Section 4 treats the expected shortfall for general elliptic linear portfolios and for the special case of Student ones. Finally, in section 5 we discuss some potential application areas.

\section{Linear Portfolio VaR with elliptic distributions}

In this section we perform a parametric analysis that relies on the assumption that the pricing function of the portfolio is linear in the risk factors. Note that parametric methods provide very fast answers which are, however, only as accurate as the underlying linearity assumption.

We will use the following notational conventions for the action of matrices on vectors: single letters $x, y, \cdots$ will denote row vectors $\left(x_{1}, \cdots, x_{n}\right),\left(y_{1}, \cdots y_{n}\right)$. The corresponding column vectors will be denoted by $x^{t}, y^{t}$, the ${ }^{t}$ standing more generally for taking the transpose of any matrix. Matrices $A=\left(A_{i j}\right)_{i, j}, B$, etc. will be multiplied in the usual way. In particular, $A$ will act on vectors by leftmultiplication on column vectors, $A y^{t}$, and by right multiplication on row vectors, $x A ; x \cdot x=x x^{t}=x_{1}^{2}+\cdots+x_{n}^{2}$ will stand for the Euclidean inner product.

A portfolio with time- $t$ value $\Pi(t)$ is called linear if its profit and loss $\Delta \Pi(t)=$ $\Pi(t)-\Pi(0)$ over a time window, $[0 \mathrm{t}]$ is a linear function of the returns $X_{1}(t), \ldots, X_{n}(t)$ 
of its constituents over the same time period:

$$
\Delta \Pi(t)=\delta_{1} X_{1}+\delta_{2} X_{2}+\ldots+\delta_{n} X_{n}
$$

This will for instance be the case for ordinary portfolios of common stock, if we use percentage returns, and will also hold to good approximation with log-returns, provided the time window $[0, \mathrm{t}]$ is small. We will drop the time $t$ from our notations, since it will be kept fixed, and simply write $X_{j}, \Delta \Pi$, etc. We also put

$$
X=\left(X_{1}, \cdots, X_{n}\right) \text {, }
$$

so that $\Delta \Pi=\delta \cdot X=\delta X^{t}$.

We now assume that the $X_{j}$ are elliptically distributed with mean $\mu$ and correlation matrix $\Sigma=A A^{t}$ :

$$
\left(X_{1}, \ldots, X_{n}\right) \sim N(\mu, \Sigma, \phi) .
$$

This means that the pdf of $\mathrm{X}$ is of the form

$$
f_{X}(x)=|\Sigma|^{-1} g\left((x-\mu) \Sigma^{-1}(x-\mu)^{t}\right),
$$

where $|\Sigma|$ stands for the determinant of $\Sigma$, and where $g: \mathbb{R}_{>0} \rightarrow 0$ is such that the Fourier transform of $g\left(|x|^{2}\right)$, as a generalized function on $\mathbb{R}^{n}$, is equal to $\phi\left(|\xi|^{2}\right)^{1}$. Assuming that $g$ is continuous, and non-zero everywhere, the Value at Risk at a confidence level of $1-\alpha$ is given by solution of the following equation:

$$
\operatorname{Prob}\left\{\Delta \Pi(t)<-V a R_{\alpha}\right\}=\alpha
$$

Here we follow the usual convention of recording portfolio losses by negative numbers, but stating the Value-at-Risk as a positive quantity of money.

In terms of our elliptic distribution parameters we have to solve the following equation:

$$
\alpha=|\Sigma|^{-1 / 2} \int_{\left\{\delta \cdot x \leq-V a R_{\alpha}\right\}} g\left((x-\mu) \Sigma^{-1}(x-\mu)^{t}\right) d x .
$$

Changing variables to $y=(x-\mu) A^{-1}, d y=|A| d x$, where $\Sigma=A^{t} A$ is a Cholesky decomposition of $A$, this becomes

$$
\alpha=\int_{\left\{\delta A \cdot y \leq-\delta \cdot \mu-V a R_{\alpha}\right\}} g\left(|y|^{2}\right) d y .
$$

Let $R$ be a rotation which sends $\delta A$ to $(|\delta A|, 0, \ldots, 0)$. Changing variables once more to $y=z R$, we obtain the equation

$$
\alpha=\int_{\left\{|\delta A| z_{1} \leq-\delta \cdot \mu-V a R_{\alpha}\right\}} g\left(|z|^{2}\right) d z .
$$

If we write that $|z|^{2}=z_{1}^{2}+\left|z^{\prime}\right|^{2}$ with $z^{\prime} \in \mathbb{R}^{n-1}$ then we have shown that :

$$
\alpha=\operatorname{Prob}\left\{\delta \cdot X<-V a R_{\alpha}\right\}=\int_{\mathbf{R}^{n-1}}\left[\int_{+\infty}^{\frac{-\delta \cdot \mu-V a R_{\alpha}}{|\delta A|}} g\left(z_{1}^{2}+\left|z^{\prime}\right|^{2}\right) d z_{1}\right] d z^{\prime} .
$$

Next, by using spherical variables $z^{\prime}=r \xi$ with $\xi \in S_{n-2}, d z^{\prime}=r^{n-2} d \sigma(\xi) d r$, we see that we have to solve for $V a R_{\alpha}$ in the equation

$$
\alpha=\left|S_{n-2}\right| \int_{0}^{+\infty} r^{n-2}\left[\int_{-\infty}^{\frac{-\delta \mu^{t}-V a R_{\alpha}}{|\delta A|}} g\left(z_{1}^{2}+r^{2}\right) d z_{1}\right] d r
$$

\footnotetext{
${ }^{1}$ One uses $\phi$ as a parameter for the class of elliptic distributions, since it is always well-defined as a continuous function: $\phi\left(|\xi|^{2}\right)$ is simply the characteristic function of an $X \sim N(0, I d, \phi)$. Note, however, that in applications we'd rather know $g$
} 
$\left|S_{n-2}\right|$ being the surface measure of the unit-sphere in $\mathbb{R}^{n-1}$ :

$$
\left|S_{n-2}\right|=\frac{2 \pi^{\frac{n-1}{2}}}{\Gamma\left(\frac{n-1}{2}\right)} .
$$

We now introduce the function

$$
\begin{aligned}
G(s) & =\frac{2 \pi^{\frac{n-1}{2}}}{\Gamma\left(\frac{n-1}{2}\right)} \int_{-\infty}^{-s}\left[\int_{0}^{+\infty} r^{n-2} g\left(z_{1}^{2}+r^{2}\right) d r\right] d z_{1} \\
& =\frac{\pi^{\frac{n-1}{2}}}{\Gamma\left(\frac{n-1}{2}\right)} \int_{s}^{-\infty} \int_{z_{1}^{2}}^{+\infty}\left(u-z_{1}^{2}\right)^{\frac{n-3}{2}} g(u) d u d z_{1},
\end{aligned}
$$

where for the second line we changed variables $u=r^{2}+z_{1}^{2}$. and replaced $z_{1}$ by $-z_{1}$. We then have proved the following result:

Theorem 2.1. Suppose that the portfolio's Profit $\&$ Loss function over the time window of interest is, to good approximation, given by $\Delta \Pi=\delta_{1} X_{1}+\delta_{2} X_{2}+\ldots+$ $\delta_{n} X_{n}$, with constant portfolio weights $\delta_{j}$. Suppose moreover that the random vector $X=\left(X_{1}, \cdots, X_{n}\right)$ of underlying risk factors follows a continuous elliptic distribution, with probability density given by $f_{X}(x)=|\Sigma|^{-1} g\left((x-\mu) \Sigma^{-1}(x-\mu)^{t}\right)$ where $\mu$ is the vector mean and $\Sigma$ is the variance-covariance matrix, and where we suppose that $g\left(s^{2}\right)$ is integrable over $\mathbb{R}$, continuous and nowhere 0 . Then the portfolio's Delta-elliptic $\operatorname{VaR} V a R_{\alpha}$ at confidence $1-\alpha$ is given by

$$
V a R_{\alpha}=-\delta \cdot \mu+q_{\alpha, n}^{g} \cdot \sqrt{\delta \Sigma \delta^{t}}
$$

where $q_{\alpha}=q_{\alpha, n}^{g}$ is the, unique, solution of the transcendental equation

$$
\alpha=G\left(q_{\alpha, n}\right) .
$$

Remark 2.2. Note that $|\delta A|$ has a clear financial interpretation, since

$$
|\delta A|=\sqrt{\delta \cdot \Sigma \cdot \delta^{t}}
$$

which is simply the portfolio's volatility, or the square of its variance.

Remark 2.3. In short-term Risk Management one can usually assume that $\mu \simeq 0$. In that case, we have that

$$
V a R_{\alpha}=\sqrt{\delta \Sigma \delta^{t}} \cdot q_{\alpha, n}^{g}
$$

which is completely analogous to the result for linear portfolios with normally distributed risk factors, except that, for example for $\alpha=0.05$, the normal quantile at $5 \%$, which is approximately 1.65 , is now replaced by the $g$-dependent constant $q_{0.05}^{g}$. The latter will have to be computed numerically, for the different $g$ 's one would like to use.

Remark 2.4. One can in fact do the integral over $z_{1}$ in (3): by Fubini,

$$
G(s)=\int_{s}^{\infty} K(s, u) g(u) d u
$$

where the kernel $K$ is given by:

$$
\begin{aligned}
K(s, u) & =\frac{1}{2}\left|S_{n-2}\right| \int_{\sqrt{s}}^{\sqrt{u}}\left(u-z_{1}\right)^{\frac{n-3}{2}} d z_{1} \\
& =\frac{1}{4}\left|S_{n-2}\right| \int_{s}^{u}(u-y)^{\frac{n-3}{2}} y^{-\frac{1}{2}} d y \\
& =\frac{1}{4}\left|S_{n-2}\right| \int_{0}^{u-s} x^{\frac{n-3}{2}}(u-x)^{-\frac{1}{2}} d x .
\end{aligned}
$$


At this stage we can the following integral from Gradshteyn and Ryzhik 4]:

$$
\int_{0}^{u} \frac{x^{\mu-1}}{(1+\beta x)^{\nu}} d x=\frac{u^{\mu}}{\mu}{ }_{2} F_{1}(\nu, \mu ; \mu+1 ;-\beta u),
$$

provided Re $\mu>0$ and $|\arg (1+\beta u)|<\pi$; cf. 4, formula 3.194(1). It follows that

$$
K(s, u)=\frac{\pi^{\frac{n-1}{2}}}{\Gamma\left(\frac{n+1}{2}\right)}(u-s)^{\frac{n-1}{2}}{ }_{2} F_{1}\left(\frac{1}{2}, \frac{n-1}{2} ; \frac{n+1}{2} ; u(u-s)\right) .
$$

However, we shall see in the example of the multi-variate $t$-distribution which we will treat next, that it can be easier to work directly with the double integral version (3) instead of with (5), (6).

2.1. The case of t-student Distributions. We now consider in detail the case where our elliptic distribution is a multivariate Student- $t$. We will, unsurprisingly, call the corresponding $V a R$ the Delta-Student $V a R$, generalizing the familiar terminology of Delta Normal VaR.

In the case of multi-variate t-student distributions, the portfolio probability density function is given by:

$$
f_{X}(x)=\frac{\Gamma\left(\frac{\nu+n}{2}\right)}{\Gamma(\nu / 2) \cdot \sqrt{|\Sigma|(\nu \pi)^{n}}}\left(1+\frac{(x-\mu)^{t} \Sigma^{-1}(x-\mu)}{\nu}\right)^{\left(\frac{-\nu-n}{2}\right)},
$$

$x \in \mathbb{R}^{n}$ and $\nu>2$. Hence $g$ is given by

$$
g(s)=C(\nu, n)(1+s / \nu)^{-\frac{(n+\nu)}{2}}, \quad s \geq 0,
$$

Where we have put

$$
C(\nu, n)=\frac{\Gamma\left(\frac{\nu+n}{2}\right)}{\Gamma(\nu / 2) \sqrt{(\nu \pi)^{n}}} .
$$

Using this $g$ in (3), we find that

$$
G(s)=\frac{\nu^{\frac{n+\nu}{2}}}{2}\left|S_{n-2}\right| C(\nu, n) \int_{s}^{\infty} I\left(z_{1}\right) d z_{1},
$$

where we have put

$$
I\left(z_{1}\right)=\int_{z_{1}^{2}}^{+\infty}\left(u-z_{1}^{2}\right)^{\frac{n-3}{2}}(\nu+u)^{-\frac{(n+\nu)}{2}} d u .
$$

The function $I\left(z_{1}\right)$ can be evaluated with the help of another one of the integrals in [4:

Lemma 2.5. (Cf. 4] ,page 314.) If $\left|\arg \left(\frac{u}{\beta}\right)\right|<\pi$, and $\operatorname{Re}\left(\nu_{1}\right)>\operatorname{Re}(\mu)>0$, then

$$
\int_{w}^{+\infty}(x-w)^{\mu-1}(\beta+x)^{-\nu_{1}} d x=(w+\beta)^{\mu-\nu_{1}} B\left(\nu_{1}-\mu, \mu\right),
$$

with $B(\alpha, \beta)$ the Euler Beta function:

$$
B(\alpha, \beta)=\frac{\Gamma(\alpha) \Gamma(\beta)}{\Gamma(\alpha+\beta)} .
$$

Using formula (91) with $\nu_{1}=\frac{(n+\nu)}{2}, \mu=\frac{n-1}{2}, \beta=\nu$, and $w=z_{1}^{2}$, and therefore, $\mu-\nu_{1}=-\frac{1+\nu}{2}$ and $-\mu+\nu_{1}=\frac{1+\nu}{2}$, we find that

$$
I\left(z_{1}\right)=\left(z_{1}^{2}+\nu\right)^{-\frac{1+\nu}{2}} B\left(\frac{1+\nu}{2}, \frac{n-1}{2}\right)
$$


We have not finished yet, since we still have to integrate over $z_{1}$ in (7). We therefore have to evaluate

$$
J(s, \nu)=\int_{-\infty}^{-s}\left(z_{1}^{2}+\nu\right)^{-\frac{1+\nu}{2}} d z_{1}
$$

Changing variable in this integral according to $u=z_{1}^{2}$, we find that

$$
J(s, \nu)=\frac{1}{2} \int_{s^{2}}^{\infty} u^{-\frac{1}{2}}(u+\nu)^{-\frac{1+\nu}{2}} d u
$$

For the latter integral, we will use another formula from [4:

Lemma 2.6. (cf. 4, formula 3.194(2)). If $\left|\arg \left(\frac{u}{\beta}\right)\right|<\pi$, and $\operatorname{Re}\left(\nu_{1}\right)>\operatorname{Re}(\mu)>0$ , then

$$
\int_{u}^{+\infty} x^{\mu-1}(1+\beta x)^{-\nu_{1}} d x=\frac{u^{\mu-\nu_{1}} \beta^{-\nu_{1}}}{\nu_{1}-\mu}{ }_{2} F_{1}\left(\nu_{1}, \nu_{1}-\mu ; \nu_{1}-\mu+1 ;-\frac{1}{\beta \cdot u}\right) .
$$

Here ${ }_{2} F_{1}(\alpha ; \beta, \gamma ; w)$ is the hypergeometric function.

In our case, $\nu_{1}=\frac{1+\nu}{2}, \mu=\frac{1}{2}, \nu_{1}-\mu=\frac{\nu}{2}, \beta=\nu^{-1}$, and $u=s^{2}$. If we replace in (12), we will obtain the following expression.

$$
J(s, \nu)=\frac{2}{\nu} s_{2}^{-\nu} F_{1}\left(\frac{1+\nu}{2}, \frac{\nu}{2} ; 1+\frac{\nu}{2} ;-\frac{\nu}{s^{2}}\right)
$$

Recalling (7), we find, after a small computation, that in the Student- $t$ case,

$$
\begin{aligned}
G(s)=G_{\nu}^{\mathrm{t}}(s) & =\frac{1}{\nu} \nu^{\frac{n+\nu}{2}}\left|S_{n-2}\right| C(\nu, n) s^{-\nu}{ }_{2} F_{1}\left(\frac{1+\nu}{2}, \frac{\nu}{2} ; 1+\frac{\nu}{2} ;-\frac{\nu}{s^{2}}\right) \\
& =\frac{1}{\nu \sqrt{\pi}}\left(\frac{\nu}{s^{2}}\right)^{\nu / 2} \frac{\Gamma\left(\frac{\nu+1}{2}\right)}{\Gamma\left(\frac{\nu}{2}\right)}{ }_{2} F_{1}\left(\frac{1+\nu}{2}, \frac{\nu}{2} ; 1+\frac{\nu}{2} ;-\frac{\nu}{s^{2}}\right) .
\end{aligned}
$$

Hence we have proved the following result on Delta-Student VaR:

Theorem 2.7. Assuming that $\Delta \Pi \simeq \delta_{1} X_{1}+\delta_{2} X_{2}+\ldots+\delta_{n} X_{n}$ with a multivariate Student-t random vector $\left(X_{1}, X_{2}, . ., X_{n}\right)$ with vector mean $\mu$, and variancecovariance matrix $\Sigma$, the linear Value-at-Risk at confidence $1-\alpha$ is given by the following formula

$$
\operatorname{VaR}_{\alpha}=-\delta \cdot \mu+q_{\alpha, \nu}^{\mathrm{t}} \cdot \sqrt{\delta \Sigma \delta^{t}},
$$

where now $s=q_{\alpha}^{t}$ is the unique positive solution of the transcendental equation

$$
G_{\nu}^{\mathrm{t}}\left(q_{\alpha . \nu}^{\mathrm{t}}\right)=\alpha
$$

with $G_{\mathfrak{t}}$ defined by 15).

Remark 2.8. Note that $q_{\alpha, \nu}^{\mathrm{t}}$ does not depend of $n$.

Hypergeometric ${ }_{2} F_{1}$ 's have been extensively studies, and numerical software for their evaluation is available in Maple and in Mathematica.

2.2. Some Numerical Result of Delta student VaR coefficient $q_{\alpha, \nu}$. In the following table, we estimate only the positive solution of $G(s)=\alpha$ for some $\nu$ Given, with the help of Mathematica 4 Software.

\begin{tabular}{|c|c|c|c|c|c|c|c|c|}
\hline$\nu$ & 2 & 3 & 4 & 5 & 6 & 7 & 8 & 9 \\
\hline$q_{0.01, \nu}$ & 6.96456 & 4.54056 & 3.74695 & 3.36493 & 3.14267 & 2.99795 & 2.89646 & 2.8214 \\
\hline$q_{0.025, \nu}$ & 4.3026 & 3.18244 & 2.77644 & 2.57058 & 2.44691 & 2.36462 & 2.3060 & 2.26216 \\
\hline$q_{0.05, \nu}$ & 2.91999 & 2.35336 & 2.13185 & 2.01505 & 1.94318 & 1.89458 & 1.85955 & 1.81246 \\
\hline
\end{tabular}




\begin{tabular}{|c|c|c|c|c|c|c|c|c|}
\hline$\nu$ & 10 & 100 & 200 & 250 & 275 & 300 & 400 & 1000 \\
\hline$q_{0.01, \nu}$ & 2.76377 & 2.36422 & 2.34135 & 2.34514 & 2.33998 & 2.33884 & 2.33571 & 2.33008 \\
\hline$q_{0.025, \nu}$ & 2.22814 & 1.98397 & 1.97189 & 1.96949 & 1.96862 & 1.9679 & 1.96591 & 1.96234 \\
\hline$q_{0.05, \nu}$ & 1.66023 & 1.66023 & 1.65251 & 1.65097 & 1.65041 & 1.64995 & 1.64867 & 1.64638 \\
\hline
\end{tabular}

Remark 2.9. Note that, we obtain practically the same result as in the case of normal distribution, when the degree of freedom of our t-student is sufficiently high ( $\nu$ near 300), as it of course should.

\section{Linear VaR With miXtures of Elliptic Distributions}

Mixture distributions can be used to model situations where the data can be viewed as arising from two or more distinct classes of populations; see also $\underline{6}$. For example, in the context of Risk Management, if we divide trading days into two sets, quiet days and hectic days, a mixture model will be based on the fact that returns are moderate on quiet days, but can be unusually large or small on hectic days. Practical applications of mixture models to compute VaR can be found in Zangari (1996), who uses a mixture normal to incorporate fat tails in VaR estimation. Here we sketch how to generalize the preceding section to the situation where the joint log-returns follow a mixture of elliptic distributions, that is, a convex linear combination of elliptic distributions.

Definition 3.1. We say that $\left(X_{1}, \ldots, X_{n}\right)$ has a joint distribution that is the mixture of $m$ elliptic distributions $N\left(\mu_{j}, \Sigma_{j}, \phi_{j}\right)^{2}$, with weights $\left\{\beta_{j}\right\}\left(\mathrm{j}=1, . ., \mathrm{m} ; \beta_{j}>0\right.$ $\left.; \sum_{j=1}^{m} \beta_{j}=1\right)$, if its cumulative distribution function can be written as

$$
F_{X_{1}, \ldots, X_{n}}\left(x_{1}, \ldots, x_{n}\right)=\sum_{j=1}^{m} \beta_{j} F_{j}\left(x_{1}, \ldots, x_{n}\right)
$$

with $F_{j}\left(x_{1}, \ldots, x_{n}\right)$ the cdf of $N\left(\mu_{j}, \Sigma_{j}, \phi_{j}\right)$.

Remark 3.2. In practice, one would usually limit oneself to $m=2$, due to estimation and identification problems; see [6].

We will suppose that all our elliptic distributions $N\left(\mu_{j}, \Sigma_{j}, \phi_{j}\right)$ admit a pdf :

$$
f_{j}(x)=\left|\Sigma_{j}\right|^{-1 / 2} g_{j}\left(\left(x-\mu_{j}\right) \Sigma_{j}^{-1}\left(x-\mu_{j}\right)^{t}\right) .
$$

The pdf of the mixture will then simp;y be $\sum_{j=1}^{m} \beta_{j} f_{j}(x)$.

Let

$$
\Sigma_{j}=A_{j}^{t} A_{j}
$$

be a Cholesky decomposition of $\Sigma_{j}$. Since integration is a linear operation, we now have to solve

$$
\alpha=\left|S_{n-2}\right| \sum_{j=1}^{m} \beta_{j}\left|\Sigma_{j}\right|^{-1 / 2} \int_{0}^{+\infty} r^{n-2}\left[\int_{-\infty}^{\frac{-\delta \cdot \mu_{j}-V a R_{\alpha}}{\left|\delta A_{j}\right|}} g_{j}\left(z_{1}^{2}+r^{2}\right) d z_{1}\right] d r
$$

to obtain $V a R_{\alpha}$. This leads to the following theorem:

\footnotetext{
$2_{\text {or }} N\left(\mu_{j}, \Sigma_{j}, g_{j}\right)$ if we parameterize elliptical distributions using $g$ instead of $\phi$
} 
Theorem 3.3. Let $\Delta \Pi=\delta_{1} X_{1}+\ldots+\delta_{n} X_{n}$ with $\left(X_{1}, \ldots, X_{n}\right)$ is a mixture of elliptic distributions, with density

$$
f(x)=\sum_{j=1}^{m} \beta_{j}\left|\Sigma_{j}\right|^{-1 / 2} g_{j}\left(\left(x-\mu_{j}\right) \Sigma_{j}^{-1}\left(x-\mu_{j}\right)^{t}\right)
$$

where $\mu_{j}$ is the vector mean, and $\Sigma_{j}$ the variance-covariance matrix of the $j$-th component of the mixture. We suppose that each $g_{j}$ is integrable function over $\mathbb{R}$, and that the $g_{j}$ never vanish jointly in a point of $\mathbb{R}^{m}$. Then the value-at-Risk, or Delta mixture-elliptic VaR, at confidence $1-\alpha$ is given as the solution of the transcendental equation

$$
\alpha=\sum_{j=1}^{m} \beta_{j} G_{j}\left(\frac{\delta \cdot \mu_{j}^{t}+V a R_{\alpha}}{\left(\delta \Sigma_{j} \delta\right)^{1 / 2}}\right),
$$

where $G_{j}$ is defined by (3) with $g=g_{j}$. Here $\delta=\left(\delta_{1}, \ldots, \delta_{n}\right)$.

Remark 3.4. In the case of a mixture of $m$ elliptic distributions the VaR will not depend any more in a simple way on the total portfolio mean and variancecovariance. This is unfortunate, but already the case for a mixture of normal distributions.

Remark 3.5. One might, in certain situations, try to model with a mixture of elliptic distributions which all have the same variance-covariance and the same mean, and obtain for example a mixture of different tail behaviors by playing with the $g_{j}$ 's. In that case the $\mathrm{VaR}$ again simplifies to: $V a R_{\alpha}=-\delta \cdot \mu+q_{\alpha} \cdot \sqrt{\delta \Sigma \delta^{t}}$, with $q_{\alpha}$ now the unique positive solution to

$$
\alpha=\sum_{j=1}^{m} \beta_{j} G_{j}\left(q_{\alpha}\right) .
$$

The preceding can immediately be specialized to a mixture of Student $\mathbf{t}$-distributions: the details will be left to the reader.

\section{Expected Shortfall For Elliptic Distributions}

Expected shortfall is a sub-additive risk statistic that describes how large losses are on average when they exceed the VaR level. Expected shortfall will therefore give an indication of the size of extreme losses when the VaR threshold is breached. We will evaluate the expected shortfall for a linear portfolio under the hypothesis of elliptically distributed risk factors. Mathematically, the expected shortfall associated with a given VaR is defined as:

$$
\text { Expected Shortfall }=\mathbb{E}(-\Delta \Pi \mid-\Delta \Pi>V a R),
$$

see for example 10. Assuming again a multivariate elliptic probability density $f(x)=|\Sigma|^{-1} g\left((x-\mu) \Sigma^{-1}(x-\mu)^{t}\right)$, the Expected Shortfall at confidence level $1-\alpha$ is given by

$$
\begin{aligned}
-E S_{\alpha} & =\mathbb{E}\left(\Delta \Pi \mid \Delta \Pi \leq-V a R_{\alpha}\right) \\
& =\frac{1}{\alpha} \mathbb{E}\left(\Delta \Pi \cdot 1_{\left\{\Delta \Pi \leq-V a R_{\alpha}\right\}}\right) \\
& =\frac{1}{\alpha} \int_{\left\{\delta x^{t} \leq-V a R_{\alpha}\right\}} \delta x^{t} f(x) d x \\
& =\frac{|\Sigma|^{-1 / 2}}{\alpha} \int_{\left\{\delta x^{t} \leq-V a R_{\alpha}\right\}} \delta x^{t} g\left((x-\mu) \Sigma^{-1}(x-\mu)^{t}\right) d x .
\end{aligned}
$$


Let $\Sigma=A^{t} A$, as before.Doing the same linear changes of variables as in section 2, we arrive at:

$$
\begin{aligned}
-E S_{\alpha} & =\frac{1}{\alpha} \int_{\left\{|\delta A| z_{1} \leq-\delta \cdot \mu-V a R_{\alpha}\right\}}\left(|\delta A| z_{1}+\delta \cdot \mu\right) g\left(\|z\|^{2}\right) d z \\
& =\frac{1}{\alpha} \int_{\left\{|\delta A| z_{1} \leq-\delta \cdot \mu-V a R_{\alpha}\right\}}|\delta A| z_{1} g\left(\|z\|^{2}\right) d z+\delta \cdot \mu .
\end{aligned}
$$

The final integral on the right hand side can be treated as before, by writing $\|z\|^{2}=$ $z_{1}^{2}+\left\|z^{\prime}\right\|^{2}$ and introducing spherical coordinates $z^{\prime}=r \xi, \xi \in S_{n-2}$, leading to:

$$
-E S_{\alpha}=\delta \cdot \mu+\frac{\left|S_{n-2}\right|}{\alpha} \int_{0}^{\infty} r^{n-2}\left[\int_{-\infty}^{\frac{-\delta \mu^{t}-V a R_{\alpha}}{|\delta A|}}|\delta A| z_{1} g\left(z_{1}^{2}+r^{2}\right) d z_{1}\right] d r
$$

We now first change $z_{1}$ into $-z_{1}$, and then introduce $u=z_{1}^{2}+r^{2}$, as before. If we recall that, by theorem 2.1

$$
q_{\alpha, n}^{g}=\frac{\delta \cdot \mu+V a R_{\alpha}}{|\delta A|}
$$

then, simply writing $q_{\alpha}$ for $q_{\alpha, n}^{g}$, we arrive at:

$$
\begin{aligned}
E S_{\alpha} & =-\delta \cdot \mu+|\delta A| \frac{\left|S_{n-2}\right|}{\alpha} \cdot \int_{q_{\alpha}} \int_{z_{1}^{2}}^{\infty} z_{1}\left(u-z_{1}^{2}\right)^{\frac{n-3}{2}} g(u) d u d z_{1} \\
& =-\delta \cdot \mu+|\delta A| \frac{\left|S_{n-2}\right|}{\alpha} \cdot \int_{q_{\alpha}^{2}}^{\infty} \frac{1}{n-1}\left(u-q_{\alpha}^{2}\right)^{\frac{n-1}{2}} g(u) d u,
\end{aligned}
$$

since

$$
\int_{q_{\alpha}}^{\sqrt{u}} z_{1}\left(u-z_{1}^{2}\right)^{\frac{n-3}{2}}=\frac{1}{n-1}\left(u-q_{\alpha}^{2}\right)^{\frac{n-1}{2}} .
$$

After substituting the formula for $\left|S_{n-2}\right|$ and using the functional equation for the $\Gamma$-function, $\Gamma(x+1)=x \Gamma(x)$, we arrive at the following result:

Theorem 4.1. Suppose that the portfolio is linear in the risk-factors $X=\left(X_{1}, \cdots, X_{n}\right)$ : $\Delta \Pi=\delta \cdot X$ and that $X \sim N(\mu, \Sigma, \phi)$, with pdf $f(x)=|\Sigma|^{-1} g\left((x-\mu) \Sigma^{-1}(x-\mu)^{t}\right)$. If we write $q_{\alpha}=$, then the expected Shortfall at level $\alpha$ is given by :

$$
E S_{\alpha}=-\delta \cdot \mu+\left|\delta \Sigma \delta^{t}\right|^{1 / 2} \cdot \frac{\pi^{\frac{n-1}{2}}}{\alpha \cdot \Gamma\left(\frac{n+1}{2}\right)} \cdot \int_{\left(q_{\alpha, n}^{g}\right)^{2}}^{\infty}\left(u-\left(q_{\alpha, n}^{g}\right)^{2}\right)^{\frac{n-1}{2}} g(u) d u
$$

4.1. Application: Student Expected Shortfall. In the case of multi-variate t-student distributions we have that $g(u)=C(\nu, n)(1+u / \nu)^{-\frac{(n+\nu)}{2}}$, with $C(\nu, n)$ given in section 2. Let us momentarily write $q$ for $q_{\alpha, \nu}^{\mathrm{t}}$. We can evaluate the integral in (19) using lemma 2.5] as follows:

$$
\begin{aligned}
& \int_{q^{2}}^{\infty}(u-q)^{\frac{n-1}{2}}\left(1+\frac{u}{\nu}\right)^{-\frac{n+\nu}{2}} d u \\
= & \nu^{\frac{n+\nu}{2}}\left(q^{2}+\nu\right)^{-\left(\frac{\nu-1}{2}\right)} B\left(\frac{\nu-1}{2}, \frac{n+1}{2}\right) .
\end{aligned}
$$

If we pose that :

$$
e s_{\alpha, \nu}=\frac{1}{\alpha \cdot \sqrt{\pi}} \frac{\Gamma\left(\frac{\nu-1}{2}\right)}{\Gamma\left(\frac{\nu}{2}\right)} \nu^{\nu / 2}\left(\left(q_{\alpha, \nu}^{\mathrm{t}}\right)^{2}+\nu\right)^{-\left(\frac{\nu+1}{2}\right)}
$$

After substitution in (19), we find, after some computations, the following result: 
Theorem 4.2. The Expected Shortfall at confidence level $1-\alpha$ for a multi-variate Student-distributed linear portfolio $\delta \cdot X$, with

$$
X \sim \frac{\Gamma\left(\frac{\nu+n}{2}\right)}{\Gamma(\nu / 2) \cdot \sqrt{|\Sigma|(\nu \pi)^{n}}}\left(1+\frac{(x-\mu)^{t} \Sigma^{-1}(x-\mu)}{\nu}\right)^{-\left(\frac{\nu+n}{2}\right)},
$$

is given by:

$$
\begin{aligned}
E S_{\alpha, \nu}^{\mathfrak{t}} & =-\delta \cdot \mu+\left|\delta \Sigma \delta^{t}\right|^{1 / 2} \cdot \frac{1}{\alpha \cdot \sqrt{\pi}} \frac{\Gamma\left(\frac{\nu-1}{2}\right)}{\Gamma\left(\frac{\nu}{2}\right)} \nu^{\nu / 2}\left(\left(q_{\alpha, \nu}^{\mathfrak{t}}\right)^{2}+\nu\right)^{-\left(\frac{\nu+1}{2}\right)} \\
& =-\delta \cdot \mu+\left|\delta \Sigma \delta^{t}\right|^{1 / 2} \cdot \frac{1}{\alpha \cdot \sqrt{\pi}} \frac{\Gamma\left(\frac{\nu-1}{2}\right)}{\Gamma\left(\frac{\nu}{2}\right)} \nu^{\nu / 2}\left(\left(\frac{\delta \cdot \mu+V a R_{\alpha}}{|\delta \Sigma \delta|^{1 / 2}}\right)^{2}+\nu\right)^{-\left(\frac{\nu+1}{2}\right)} \\
& =-\delta \cdot \mu+e s_{\alpha, \nu} \cdot\left|\delta \Sigma \delta^{t}\right|^{1 / 2}
\end{aligned}
$$

The Expected Shortfall for a linear Student portfolio is therefore given by a completely explicit formula, once the VaR is known. Observe that, as for the VaR, the only dependence on the portfolio dimension is through the portfolio mean $\delta \cdot \mu$ and the portfolio variance $\delta \Sigma \delta^{t}$.

With the help of Matlab we obtain the the following table of values of $e_{\alpha, \nu}$ :

\begin{tabular}{|c|c|c|c|c|c|c|c|c|}
\hline$\nu$ & 2 & 3 & 4 & 5 & 6 & 7 & 8 & 9 \\
\hline$e s_{0.01, \nu}$ & 5.5722 & 5.9309 & 5.7879 & 5.4555 & 5.0799 & 4.7160 & 4.3819 & 4.0818 \\
\hline$e s_{0.025, \nu}$ & 8.6113 & 7.6777 & 6.8216 & 6.0676 & 5.4326 & 4.9032 & 4.4601 & 4.0862 \\
\hline$e s_{0.05, \nu}$ & 11.7123 & 9.0750 & 7.4966 & 6.3797 & 5.5457 & 4.9007 & 4.3880 & 3.9711 \\
\hline
\end{tabular}

\begin{tabular}{|c|c|c|c|c|}
\hline$\nu$ & 10 & 100 & 200 & 250 \\
\hline$e s_{0.01, \nu}$ & 3.8135 & 0.5157 & 0.2644 & 0.2086 \\
\hline$e s_{0.025, \nu}$ & 3.7675 & 0.4577 & 0.2313 & 0.1854 \\
\hline$e s_{0.05, \nu}$ & 3.6257 & 0.4073 & 0.2050 & 0.1642 \\
\hline
\end{tabular}

4.2. ES for Elliptic distribution Mixtures. The preceding results can, as before, easily be generalized to mixtures of elliptic distributions. Details will be left to the reader.

\section{Some Areas of Applications}

In this section, we survey some areas for applications of linear portfolios that exist in the financial literature. We will discuss 4 examples:

- Delta-approximation of a derivatives portfolio.

- Linear approximation of an equity portfolio

- Businesses as portfolios of business units

- Incremental VaR

5.1. Delta Approximation of a Portfolio. Suppose that we are holding a portfolio of derivatives depending on $n$ underlying assets $X^{(1)}, X^{(2)}, \ldots, X^{(n)}$ with elliptically distributed log-returns $r_{j}$ (over some fixed time-window). The portfolio's present value $V$ will in general be some complicated non-linear function of the $X_{i}$ 's. To obtain a first approximation of its VaR, we simply approximate the present Value $\mathrm{V}$ of the position using a first order Taylor expansion:

$$
V(X+\Delta X) \approx V(X)+\sum_{i=1}^{n} \frac{\partial V}{\partial X^{(i)}} \Delta X_{i}
$$


¿From this, we can then approximate the profit/loss function as

$$
\Delta V=V(X+\Delta X)-V(X) \approx \sum_{i=1}^{n} \delta_{i} r^{(i)}=\delta \cdot r,
$$

where we put $r=\left(r^{(1)}, \ldots, r^{(n)}\right)$ and $\delta=\left(\delta_{1}, \ldots, \delta_{n}\right)$ with $\delta_{i}=X^{(i)} \frac{\partial V}{\partial X^{(i)}}$. The entries of the $\delta$ vector are called the "delta equivalents " for the position, and they can be interpreted as the sensitivities of the position with respect to changes in each of the risk factors. For more details see [6, where a multi-variate normal distribution for the $r_{i}$ 's is assumed. The discussion there generalizes straightforwardly to the elliptic case, where the present paper's results can be used.

5.2. Portfolios of Equities. A special case of the preceding is that of an equity portfolio, build of stock $S_{1}, \cdots, S_{n}$ with $\log$-returns $r_{1}(t), \cdot, r_{n}(t)$. In this case,

$$
\begin{aligned}
\Pi(t)-\Pi(0) & =\sum_{i=1}^{n} w_{i} S_{i}(0)\left(S_{i}(t) / S_{i}(0)-1\right) \\
& \approx \sum_{i=1}^{n} w_{i} S_{i}(0) r_{i}(t),
\end{aligned}
$$

where this approximation will be good if the $r_{i}(t)$ are small.

5.3. Businesses as Linear Portfolios of Business Units. An interesting way of looking upon an big enterprize, e.g. a multi-national or a big financial institution, is by considering it as a sum of its individual business units, cf. Dowd [7]. If $X_{j}$, is the the variation of price or of profitability of business unit $j$ in one period, then the variation of price of the agglomerate in the same period will be

$$
\Delta \Pi=X_{1}+\cdots+X_{n} .
$$

The entire institution is therefore modelled by a linear portfolio, with $\delta=(1,1, \ldots, 1)$, to which the results of this paper can be applied, if we model the vector of individual price variations by a multi-variate elliptic distribution. VaR, incremental VaR (see below) and Expected Shortfall will be relevant here. For more details see Dowd [7, chapter XI .

5.4. Incremental VaR. Incremental VaR is defined in $[6]$ as the statistic that provides information regarding the sensitivity of VaR to changes in the portfolio holdings. It therefore gives an estimation of the change in VaR resulting from a risk management decision. Results from [10] for incremental VaR with normally distributed risk-factors generalize straightforwardly to elliptically distributed ones: if we denote by $I V a R_{i}$ the incremental VaR for each position in the portfolio, with $\theta_{i}$ the percentage change in size of each position,then the change in VaR will be given by

$$
\Delta V a R=\sum \theta_{i} I V a R_{i}
$$

By using the definition of $I V a R_{i}$ as in [6] (2001), we have that

$$
I V a R_{i}=\omega_{i} \frac{\partial V a R}{\partial \omega_{i}}
$$

with $\omega_{i}$ is the amount of money invested in instrument $i$. In the case of an equity portfolio in the elliptically distributed assets, we have seen that, assuming $\mu=0$,

$$
V a R_{\alpha}=-q_{\alpha, n}^{g} \sqrt{\delta \Sigma \delta^{t}}
$$

We can then calculate $I V a R_{i}$ for the i-th constituent of portfolio as

$$
I V a R_{i}=\omega_{i} \frac{\partial V a R}{\partial \omega_{i}}=\omega_{i} \gamma_{i}
$$


with

$$
\gamma=-q_{\alpha, n}^{g} \frac{\Sigma \omega}{\sqrt{\delta \Sigma \delta^{t}}} .
$$

The vector $\gamma$ can be interpreted as a gradient of sensitivities of VaR with respect to the risk factors. This is the same as in [6], except of course that the quantile has changed from the normal one to the one associated to $g$.

\section{CONCLUSION}

In this paper we have shown how to reduce the estimation of Value-at-Risk for linear elliptic portfolios to the evaluation of one dimensional integrals which, for the special case of a Student distribution, can be explicitly evaluated in terms of a hypergeometric function. We indicated how to extend these to the case of mixtures of elliptic distributions. We have also given a similar, but simpler, integral formula for the expected shortfall of such portfolios which, again, can be completely evaluated in the Student case. We finally surveyed some potential application areas. 


\section{REFERENCES}

[1] Albenese, C., and L.Seco : "Harmonic Analysis in Value-at-Risk Calculations" , Revista Matemaatica Ibero Americana 17 (2), 2001.

[2] Duffie, D., and J.Pan : An overview of Value at Risk, J. Derivatives 4(3), 7-49.

[3] P.Embrechts , A.McNeil and D.Strauman (1999) Correlation and dependance in Risk Management Properties and Pitfalls. In M.A.H. Dempster, editor, Risk Management : Value at Risk and Beyond, pages 176-223. Cambridge University Press, 1999.

[4] I.S Gradshteyn, I.M. Ryzhik : Table of integrals, series, and products ( 2000) Editor Alan Jeffrey

[5] Glasserman, P. , Heidelberger, P. and Shahabuddin : Portfolio Value-at-Risk with Heavy Yailed Risk Factors. Mathematical Finance, 12 (3): 239-269, July 2002.

[6] Jorge Mina and Jerry Yi Xiao (2001), Return to Riskmetrics: The Evolution of the standard (www.riskmetrics.com). 2001 RiskMetrics Group Inc.

[7] Kevin Dowd,(1998) Beyond Value-at-Risk: the new science of risk Management (Wiley series in Frontiers in Finance).

[8] Lopez, J.A. and Walter,C.A.(2000). Evaluating covariance matrix Forecastsin a Value-at-Risk Framework, FRBSF working paper 2000-21, Federal Reserve Bank of San Francisco

[9] Kotz, Balahskrinan, (2001) Continuous Multivariate Distributions

[10] J.Sadefo-Kamdem ,(2003):VaR Estimation for Portfolio of Securities with joint elliptic distribution log-Returns. Working paper

[11] Yasuhiro and Toshinao Yoshiba, (2002): On the validity of Value-at-Risk: Comparative analysis with Expected shortfall

[12] Zangari, P. (1996). An Improved Methodology for Measuring VaR, RiskMetrics Monitor, 2nd quarter pp.7-25. http://www.riskmetrics.com/research/journals 